\title{
Spatio-Temporal Dynamics of Carrier Capture Processes: Simulation of Optical Signals
}

\author{
F. Lengers*, R. Rosati, T. Kuhn and D.E. Reiter \\ Institut für Festkörpertheorie, Universität Münster, Wilhelm-Klemm-Str. 10, 48149 Münster, Germany \\ We perform simulations of time-resolved optical experiments of carrier-capture processes in a quantum wire- \\ dot system. Scattering of charge carriers with optical phonons of the quantum wire can result in transitions from \\ the continuum states of the quantum wire to discrete states of the quantum dot. We treat the scattering of carriers \\ with optical phonons within a Lindblad single-particle approach. By considering the coupling of carriers to a light \\ field we are able to simulate pump-probe experiments which are shown to be capable of measuring the captured \\ populations. We further discuss the influence of the Coulomb interaction on the obtained spectra.
}

DOI: 10.12693/APhysPolA.132.372

PACS/topics: quantum dot, carrier capture, optical signals

\section{Introduction}

Carrier capture processes occur frequently in several semiconductor structures, e.g., scattering of carriers from the wetting layer to quantum dots [1-6], from a quantum wire to the embedded quantum dot [7-9], or from monolayers of transition metal dichalcogenides (TMDCs) to discrete states created by strain effects $[10,11]$. All those processes happen on subpicosecond time scales and on nanometric length scales and therefore are of genuinely quantum mechanical nature. To monitor those local and ultrafast processes one can use time resolved pump-probe experiments. In this paper we study theoretically the optical signals of spectrally and temporally resolved pumpprobe experiments of the carrier capture process from a semiconductor quantum wire (QWR) to an embedded semiconductor quantum dot (QD). We study the optical signals with and without Coulomb interaction and analyze their temporal behavior. Furthermore we describe how the captured population of the QD levels can be extracted from the optical signals. By comparing those results to the simulated population dynamics we show that the capture dynamics can be reconstructed by the optical measurements.

In order to calculate the carrier dynamics we use the Lindblad single-particle (LSP) equation of motion introduced in Ref. [12]. This LSP approach is a proper tailoring of an alternative Markov approach [13] and its consequent single-particle equation of motion [14] which is closed in the density matrix and immune to instabilities while being able to fully capture the spatio-temporal evolution of the carrier dynamics [15-17].

Previously, a spatially resolved description of the problem was performed with quantum kinetic equations explicitly taking into account carrier-phonon correlati-

\footnotetext{
* corresponding author; e-mail: Frank.Lengers@uni-muenster.de
}

ons [18-20], which leads to a very demanding calculation. In contrast, the LSP equation of motion is closed in the single-particle density matrix and thus computationally much lighter. A comparison of the two methods showed that the LSP approach reproduces the essential features displayed by quantum kinetic studies [12]. Due to the reduced computational effort we are now able to simulate time-resolved pump-probe experiments, which usually requires to run the program multiple times [21], very efficiently.

\section{Theory}

We model the semiconductor QWR as a parabolic twoband model with the effective masses of GaAs $\left(m_{e}^{*}=\right.$ $0.067 m_{0}, m_{h}^{*}=0.45 m_{0}$ with the free electron mass $m_{0}$ ). The QWR is cylindrical with a $100 \mathrm{~nm}^{2}$ cross-section and we consider the lowest lying, transverse subband assuming infinitely high confinement potentials. The QD is modelled as an effective potential entering the Schrödinger equation of the envelope function

$$
\left(-\frac{\hbar^{2}}{2 m_{e(h)}^{*}} \frac{\partial^{2}}{\partial z^{2}}+V_{e(h)}^{Q D}(z)\right) \Psi_{e(h)}(z)=\epsilon^{e(h)} \Psi_{e(h)}(z) .
$$

Throughout the paper we use $V_{e(h)}^{Q D}=-V_{0}^{e(h)} \operatorname{sech}(z / a)$ with $\frac{3}{2} V_{0}^{h}=V_{0}^{e}=-30 \mathrm{meV}$ and $a=4 \mathrm{~nm}$. The numerically estimated solutions of Eq. (1) are used as a basis for the simulation and to distinguish bound states $(\epsilon<0)$ and delocalized states $(\epsilon>0)$. The system under study has one bound state in the conduction band at $\epsilon_{1}^{e}=-14.7 \mathrm{meV}$ and two bound states in the valence band at $\epsilon_{1}^{h}=-14.1 \mathrm{meV}$ and $\epsilon_{2}^{h}=-5.1 \mathrm{meV}$.

Electron-hole pairs are excited by an electric field treated within the dipole and rotating wave approximation. The spacial localization of the laser pulse is considered by the carrier-light coupling elements $E_{n, l}(t)=$ $M\langle n|E(z, t)| l\rangle$ between the hole state $|l\rangle$ and the electron state $|n\rangle$, where $M$ is the bulk dipole moment in the direction of the field taken as a constant. 
To include excitonic effects we also take into account the attractive electron-hole Coulomb interaction within the mean-field approximation. We only consider low carrier densities so that Coulomb-induced energy renormalizations or local potentials can safely be neglected.

The carriers are coupled to phonons and since we consider a system in which the energetic separation of localized and delocalized states is relatively high (tens of meV), we include only longitudinal optical (LO) phonons of bulk GaAs with an energy $\hbar \omega_{L O}=36.4 \mathrm{meV}$. The coupling matrix element is given by the Fröhlich coupling.

While the carrier-carrier interaction in mean-field approximation and the carrier-field interaction are treated exactly within the equations of motion as in Ref. [18, 20], the carrier-phonon coupling is treated by LSP equations of motion as in Ref. [12] where the phonons are approximated as a bath which is justified for the low excited densities considered here.

\section{Results}

The electric field takes the form $\boldsymbol{E}(z, t)=\boldsymbol{E}_{1}(z, t)+$ $\boldsymbol{E}_{2}(z, t-\tau)$ with pump pulse $\boldsymbol{E}_{1}$ and probe pulse $\boldsymbol{E}_{2}$. Both take the form

$$
\boldsymbol{E}_{i}(z, t)=\boldsymbol{E}_{i} \exp \left(-\frac{t^{2}}{2 \sigma_{i}^{2}}-\mathrm{i} \omega_{L} t-\frac{\left(z-z_{0, i}\right)^{2}}{2 \Delta z_{i}^{2}}\right) .
$$

The pump pulse is focused around $z_{0,1}$ away from the QD (located at $z=0$ ) with a broadening of $\Delta z_{1}=10 \mathrm{~nm}$ and a pulse length of $\sigma_{1}=50 \mathrm{fs}$, mimicking, e.g., a localized excitation through the tip of a near-field microscope. The probe pulse is centered around the QD $\left(z_{0,2}=0 \mathrm{~nm}\right)$ with a broadening of $\Delta z_{2}=80 \mathrm{~nm}$ and a pulse length of $\sigma_{2}=10 \mathrm{fs}$. Both pulses have the excess energy $\hbar \omega_{L}-E_{\text {gap }}=20 \mathrm{meV} \approx \epsilon_{1}^{e}+\hbar \omega_{L O}$ with $\epsilon_{1}^{e}$ being the lowest electronic energy given by Eq. (1). We extract the polarization in direction of the probe field $\boldsymbol{E}_{2}$ by using a Fourier decomposition [21]. From this, the differential absorption spectrum $\Delta \alpha=\alpha-\alpha_{0}$ is calculated as difference of absorption spectra with $(\alpha)$ and without $\left(\alpha_{0}\right)$ pump pulse. In order to excite low densities we set the Rabi frequency of the pump pulse to $\Omega_{R}^{\text {pump }}=\hbar^{-1} \boldsymbol{M} \cdot \boldsymbol{E}_{1}=10^{-4} \mathrm{ps}^{-1}$ and for the probe pulse to $\Omega_{R}^{\text {probe }}=10^{-6} \mathrm{ps}^{-1}$.

\subsection{Linear absorption}

We start by analyzing the linear absorption spectra of the system with and without Coulomb interaction which are shown in Fig. 1. For $E-E_{g a p}>0$ one can see the QWR continuum contributions in both cases. Without the Coulomb interaction there is only one other peak visible at $\hbar \omega_{b s}=E-E_{g a p} \approx-29 \mathrm{meV}$ which can be directly identified as the transition between the lowest lying bound states of electrons and holes. The dipole matrix element for the transition from $\epsilon_{2}^{h}$ to $\epsilon_{1}^{e}$ vanishes and therefore this transition is not visible.

With the Coulomb interaction the spectrum is dominated by the peak at $-21 \mathrm{meV}$ which stems from the QWR

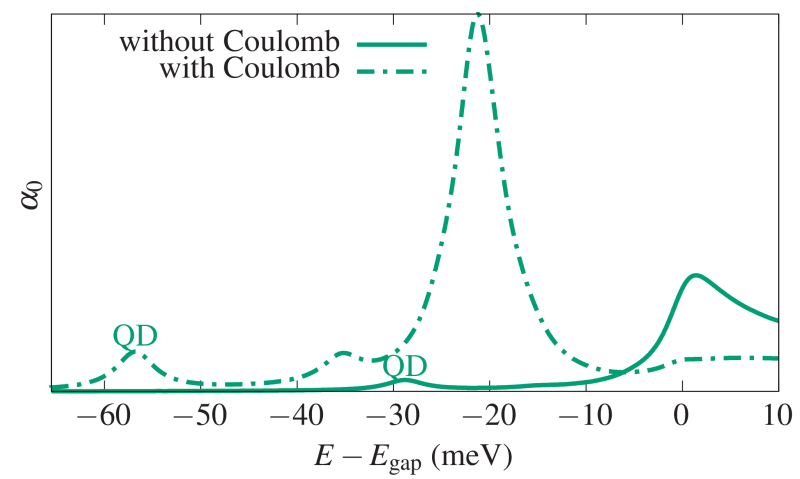

Fig. 1. Linear absorption spectra with and without Coulomb interaction.

exciton. The lowest lying peak at $-57 \mathrm{meV}$ originates again from the lowest lying electron and hole states and constitutes the QD exciton.

\subsection{Without Coulomb interaction}

We first consider the case without the Coulomb interaction. The wave packet is excited $50 \mathrm{~nm}$ away from the QD. The calculated spectra are shown in Fig. 2. Be-

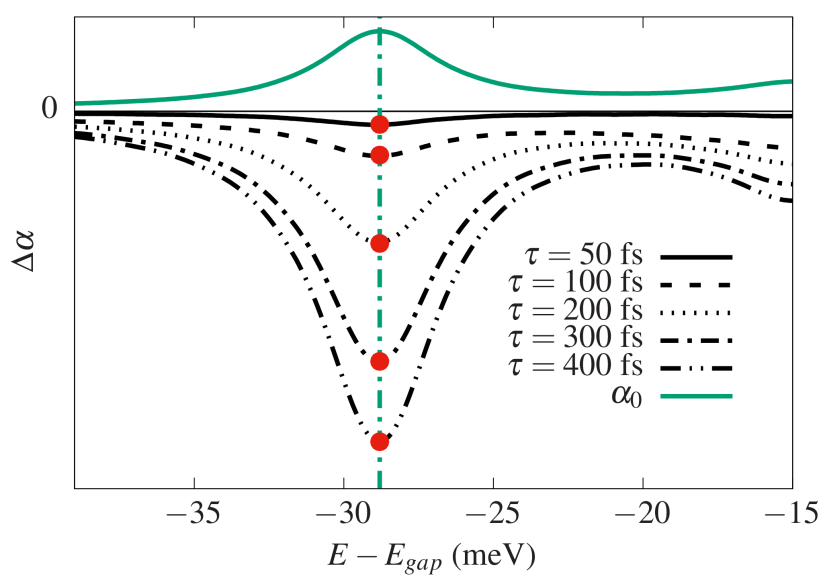

Fig. 2. Differential absorption for an excitation $50 \mathrm{~nm}$ away from the QD for different delay times $\tau$ and the absorption spectrum without the pump pulse $\left(\alpha_{0}\right.$, green line). Note that $\alpha_{0}$ has been scaled with respect to the other curves. The position of resonance is marked as a dash-dotted line. Red points depict the minimal value of $\Delta \alpha$.

cause we are interested in the occupation of the QD states, we focus on the lowest absorption peak at $\hbar \omega_{b s} \approx$ $-29 \mathrm{meV}$ originating from the QD transition. The top curve displays the absorption spectrum $\alpha_{0}$ without pump pulse, the other curves show the differential absorption spectrum $\Delta \alpha$ for different values of the delay time $\tau$ (plotted on an enlarged scale compared to $\alpha_{0}$ ). For increasing delay $\tau$ one observes that the differential absorption spectra become increasingly negative. This can be attributed to an increasing Pauli blocking in the bound states. The Pauli blocking can be attributed solely to the bound state of the conduction band, because the holes travel 


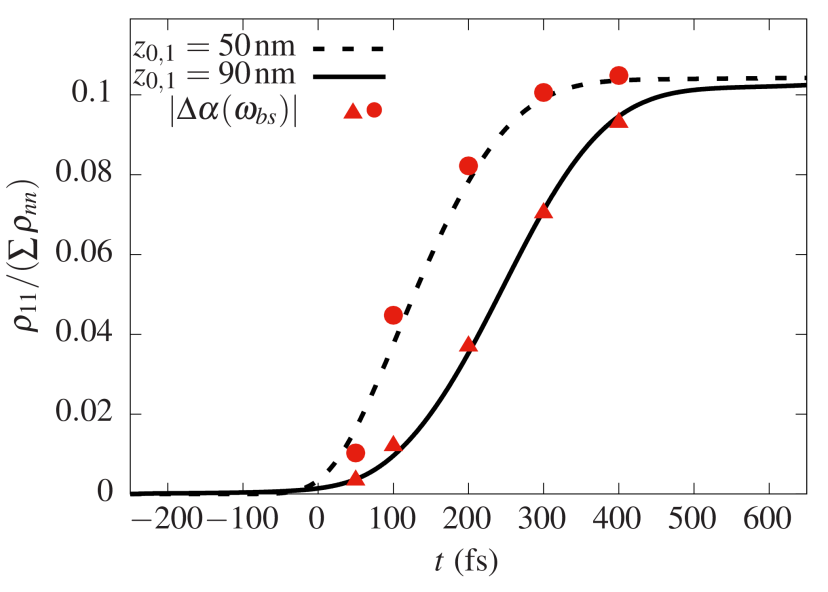

Fig. 3. Population dynamics of the electronic bound state for $z_{0,1}=50 \mathrm{~nm}$ (dashed line) and $z_{0,1}=90 \mathrm{~nm}$ (solid line) together with the scaled, extracted values of the absorption spectra from Fig. 2 (red circles and triangles, respectively).

much slower and therefore do not reach the QD within our time window. Additionally the holes are excited offresonantly with respect to the transition to bound states, resulting in a negligible capture rate. Therefore we will restrict the discussion to the electronic bound state in the following.

We now extract the increasing peak amplitude of the absorption spectra as a function of time delay (red points in Fig. 2) and compare them to the dynamics of the bound state population in Fig. 3. The extracted values are scaled by a common constant to match the population dynamics. As can be seen in Fig. 3, the dynamics of the bound state can be reconstructed very well from the pump-probe simulation.

We further look at the capture dynamics and optical signals, when the wave packet starts further away from the $\mathrm{QD}$ at $z_{0,1}=90 \mathrm{~nm}$. Because now the wave packet needs more time to reach the QD, the capture is delayed and starts approximately 115 fs later than in the previous case, underlining the local nature of the capture process [12]. Also in this case, the capture dynamics is well reproduced by the optical signals (red triangles).

\subsection{With Coulomb interaction}

Now we include Coulomb interaction. An example of the resulting spectrum is shown in Fig. 4 together with the absorption spectrum without the pump pulse. Here one ends up with a dispersive line shape of the spectrum due to a renormalization of the exciton binding energy. Such ultrafast renormalizations have indeed been observed in two-color pump-probe experiments on a single self-assembled QD [22, 23]. The observed spectrum now consists of two contributions. The first one is the dispersive line shape which, for small renormalizations (due to small excited densities), is antisymmetric around the ground state exciton energy. Superimposed is a reduction due to the Pauli blocking such that the spectrum is slightly shifted to negative values of $\Delta \alpha$. To extract the Pauli blocking, we integrate over a symmetric region $E_{e x} \pm 9 \mathrm{meV}$ around the exciton ground state energy $E_{e x}$ and put a filter $\exp \left(-\frac{\left(E-E_{e x}\right)^{2}}{2 \Delta E^{2}}\right)$ with $\Delta E=3.5 \mathrm{meV}$ on the spectrum. The extracted data are again compared to the population dynamics for two different excitation locations in Fig. 5. As one can see, the extracted values fit reasonably well with the captured density. The differences can be attributed to the integration over a region which is affected by other dispersive lines due to other excitons (see Fig. 1). Also with the Coulomb interaction, the capture happens locally and the optical signals reflect the delayed capture when the wave packet is excited further away from the QD.

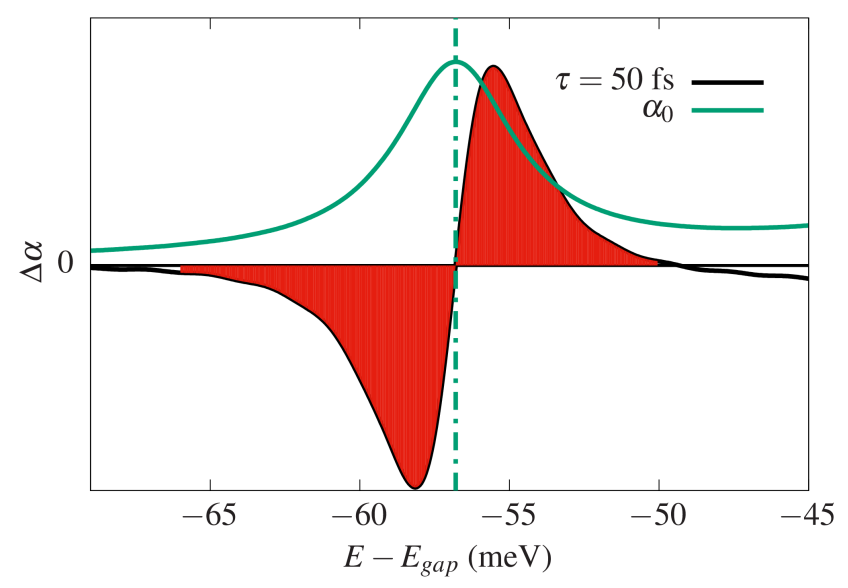

Fig. 4. Differential absorption spectra for a time delay of $50 \mathrm{fs} . \alpha_{0}$ is shown in green and scaled. The position of resonance is marked by a dash-dotted line. The integration range is indicated as red area.

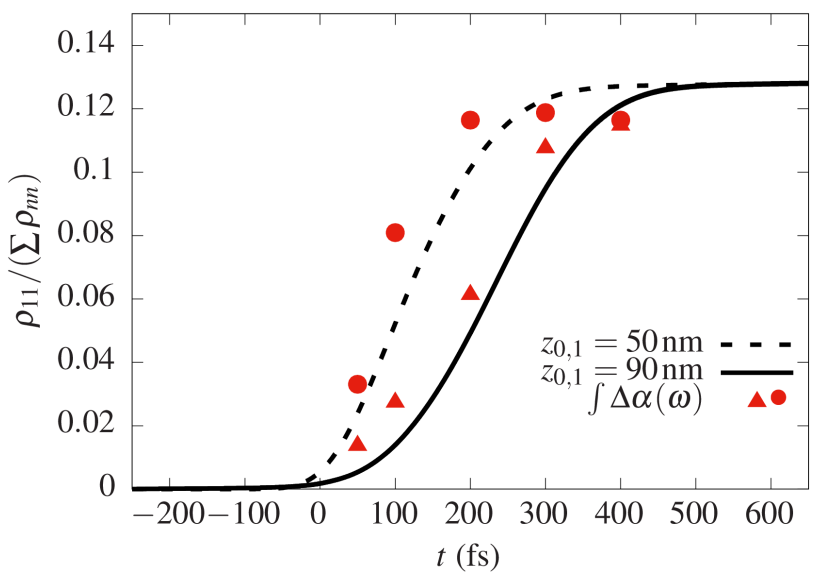

Fig. 5. As in Fig. 3, but with Coulomb interaction.

\section{Conclusions}

We have simulated optical experiments to measure carrier capture processes in a QWR-QD system. We have used the recently developed LSP approach to treat the 
phonon-induced capture process and simulated pumpprobe experiments under consideration of carrier-light and carrier-carrier interaction. The computationally light LSP approach made it possible to simulate the experiments very efficiently. We have shown that pumpprobe signals are capable of monitoring the population dynamics of the electron bound state. By varying the distance of the excited wave packet to the $\mathrm{QD}$, the optical signals show that the capture indeed happens locally.

\section{References}

[1] R. Ferreira, G. Bastard, Appl. Phys. Lett. 74, 2818 (1999).

[2] I. Magnusdottir, S. Bischoff, A.V. Uskov, J. Mørk, Phys. Rev. B 67, 205326 (2003).

[3] A. Markus, A. Fiore, Phys. Status Solidi A 201, 338 (2004).

[4] T.R. Nielsen, P. Gartner, F. Jahnke, Phys. Rev. B 69, 235314 (2004).

[5] J. Seebeck, T.R. Nielsen, P. Gartner, F. Jahnke, Phys. Rev. B 71, 125327 (2005).

[6] S. Trumm, M. Wesseli, H.J. Krenner, D. Schuh, M. Bichler, J.J. Finley, M. Betz, Appl. Phys. Lett. 87, 153113 (2005).

[7] G. Schedelbeck, W. Wegscheider, M. Bichler, G. Abstreiter, Science 278, 1792 (1997).

[8] C. Lienau, V. Emiliani, T. Guenther, F. Intoni, T. Elsaesser, R. Nötzel, K.H. Ploog, Phys. Status Solidi A 178, 471 (2000).

[9] W. Wegscheider, G. Schedelbeck, G. Abstreiter, M. Rother, M. Bichler, Phys. Rev. Lett. 79, 1917 (1997).
[10] J. Feng, X. Qian, C. Huang, J. Li, Nat. Photon. 6, 866 (2012).

[11] J. Kern, I. Niehues, P. Tonndorf, R. Schmidt, D. Wigger, R. Schneider, T. Stiehm, S. Michaelis de Vasconcellos, D.E. Reiter, T. Kuhn, R. Bratschitsch, Adv. Mater. 28, 7101 (2016).

[12] R. Rosati, D.E. Reiter, T. Kuhn, Phys. Rev. B 95 165302 (2017).

[13] D. Taj, R.C. Iotti, F. Rossi, Eur. Phys. J. B 72, 305 (2009).

[14] R. Rosati, R.C. Iotti, F. Dolcini, F. Rossi, Phys. Rev. B 90, 125140 (2014).

[15] R. Rosati, F. Rossi, Appl. Phys. Lett. 103, 113105 (2013).

[16] R. Rosati, F. Rossi, Phys. Rev. B 89, 205415 (2014).

[17] R. Rosati, F. Dolcini, F. Rossi, Appl. Phys. Lett. 106, 243101 (2015).

[18] M. Glanemann, V.M. Axt, T. Kuhn, Phys. Rev. B 72, 045354 (2005).

[19] D. Reiter, M. Glanemann, V.M. Axt, T. Kuhn, Phys. Rev. B 73, 125334 (2006).

[20] D. Reiter, M. Glanemann, V.M. Axt, T. Kuhn, Phys. Rev. B 75, 205327 (2007).

[21] L. Seidner, G. Stock, W. Domcke, J. Chem. Phys. 103, 3998 (1995).

[22] F. Sotier, T. Thomay, T. Hanke, J. Korger, S. Mahapatra, A. Frey, K. Brunner, R. Bratschitsch, A. Leitenstorfer, Nat. Phys. 5, 352 (2009).

[23] J. Huneke, I. D'Amico, P. Machnikowski, T. Thomay, R. Bratschitsch, A. Leitenstorfer, T. Kuhn, Phys. Rev. B 84, 115320 (2011). 